\title{
Advanced Shape Memory Technology for Comfort Fitting
}

\author{
Tao Xi Wang ${ }^{1,}$, , Hong Mei Chen², b , Wei Min Huang ${ }^{1, c}$ \\ ${ }^{1}$ School of Mechanical and Aerospace Engineering, Nanyang Technological University, 50 \\ Nanyang Avenue, 639798, Singapore \\ ${ }^{2}$ College of Chemistry and Materials Science, Sichuan Normal University, Chengdu 610066, \\ People's Republic of China
}

aWA0003XI@e.ntu.edu.sg, b21815597@qq.com, ‘mwmhuang@ntu.edu.sg

\begin{abstract}
Keywords: Shape memory technology, Shape memory material, Comfort fitting, Working mechanism, Shape memory effect.
\end{abstract}

\begin{abstract}
Nowadays, more and more people are keening to invest in their own personal fitness. Instead of producing customized products according to the actual dimensions of individuals, which could be highly expensive and complicate, advanced shape memory technology could be utilized to achieve one-size-for-all in a cost effective manner using well-established existing technologies. In this paper, first the concept of advanced shape memory technology is briefly introduced, together with the working principle. Some typical applications are presented here to demonstrate the potential benefits of this approach.
\end{abstract}

\section{Introduction}

Nowadays, more and more people are keening to invest in their own personal fitness. As a typical example, mis-fitting between the foot and footwear impairs foot function and may result in undue pressure on the foot from tightly fitting footwear or unwanted friction from loosely fitting footwear [1]. For instance, CrocsTM is soft, comfortable, lightweight and odor-resistant, and it is now very much popular within the young generation. However, in term of fitting, in such shoes it is only limited to within the contact interface between the underneath of foot and top of sole. There might be still indeed a large empty space between the foot and inner part of the shoes for most of the people, since the shoes are produced according standard dimensions. Hence, the foot inside is prone to moving, which increases the risk of, for instance, foot injury. It is a general trend at this moment that consumers want more comfort and functionality from their footwear [2].

Although customized shoes might be fabricated via, for instance, 3D printing for better fitting [3, 4], it mostly requires a tedious process of 3D foot scanning plus a still expensive fabrication process of printing. Such a type of one-to-one customized fabrication is actually a natural extension of tailored manufacturing in the past, which is opposite to mass fabrication for lowered cost but with minimum consideration for comfort fitting.

It can be concluded that a cost effective approach to realize comfort fitting, not only for shoes, but also for a range of other personal items, is highly in demand at present [5].

In this paper, first we introduce the concept of advanced shape memory technology and its working principle, and then we present a few typical applications for comfort fitting.

\section{Advanced Shape Memory Technology}

The shape memory effect refers to the capability of a material to recover its original shape only when the right stimulus is applied [6-9]. Such a shape recovery phenomenon is different from the shape change effect, in which a material responses to the applied stimulus in a linear or nonlinear manner with or without a hysteresis $[10,11]$. A higher energy barrier between the deformed shape, which is called the temporary shape, and the original shape, which is permanent, is the fundamental reason behind the shape memory effect $[12,13]$. 
As well explained in [14], the advanced shape memory technology is an extension of the shape memory technology, which is mainly meant for a particular type of shape memory material, namely, shape memory alloys, and their applications in the past when it was coined.

Advanced shape memory technology includes the following three parts [14],

- The techniques to enable the shape memory effect in conventional materials. So that it is not limited to shape memory alloys only, but all types of shape memory materials, even those conventional materials, such as many engineering polymers $[13,15,16]$;

- The techniques to design/tailor the shape memory function of a material to meet the requirement(s) of a particular application [12];

- The techniques to optimize the shape memory performance [17].

As summarized in [12], there are three basic working mechanisms to realize the shape memory effect in polymeric materials. From applications point of view with particular concerns for comfort fitting, there are two parameters of our interest, namely, the shape fixity ratio and shape recovery ratio. Both ratios can be tailored by various approaches. Of course, the working temperature is probability the most important parameter for consideration.

We have demonstrated that some commercially available polymeric materials can meet most requirements without any modification or with only some minor modifications [5]. On the other hand, we can develop new polymeric materials particularly for comfort fitting purpose with tailored thermo-mechanical properties, such as body-temperature programming to fix the temporary shape and rubber-like elasticity at both high and low temperatures.

\section{Typical Applications}

In terms of fitting based on the advanced shape memory technology, in addition to the following apparent advantages,

- Rapid fitting;

- Repeatable fitting;

- Comfort fitting;

- Convenient fitting;

it is also a low cost approach as it can be fabricated using conventional and well-established existing manufacturing techniques, and thus, naturally it becomes affordable to everyone.

Apart from shape memory comfort fitting shoes based on elastic polymeric foam as reported in [18], Fig. 1 demonstrates the concept of comfort fitting sock-shoes. In Fig. 1(a), the left piece is a piece of normal sock, while the right one is a piece of shape memory sock-shoe, which is actually modified from a piece of sock just like the left one (only in a different colour). After being heated to above $70{ }^{\circ} \mathrm{C}$ using an oven or a hairdryer, the sock-shoe is soft and elastic, even after cooling back to body temperature. Hence, it can be worn as normal elastic socks without the worry of your bare foot being over-heated. It is able to comfort-fits anyone's foot just like normal socks, as shown in Fig. 1(b). However, unlike normal socks, which are highly elastic, but too soft, so that they cannot provide enough protection to our foot, our shape memory sock-shoes become stiff after further prolonged cooling for solidification. Thus, they are able to provide protection function as shoes, while they are still elastic, so that you can wear/take off them easily again and again. As we can see, the sock-shoe maintains the temporary shape as shown in Fig. 1(c), unless it is heated for another cycle of fitting.

Some other typical applications that we have worked out are presented in Fig. 2, including handle, handle wrapper, fingerless glove and elbow band.

\section{Conclusions}

We have seen more and more demands for personalized items. The advanced shape memory technology provides a cost-effective approach to meet such needs. Therefore, instead of looking for the right item which comfort-fits an individual, now we are able to ensure that any item will fit an individual instantly. 


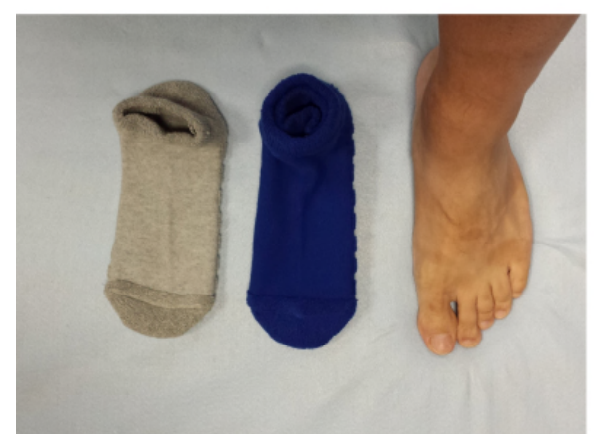

(a) Shape memory sock-shoe (middle, as received)
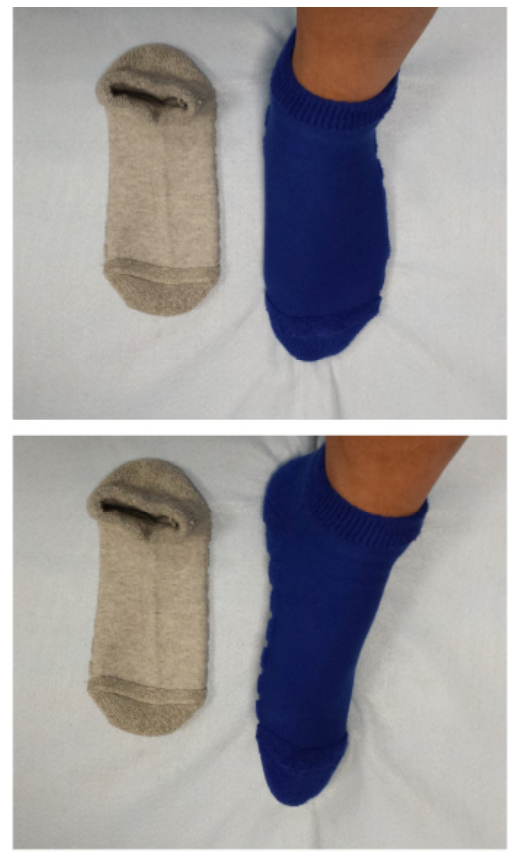

(b) Fitting
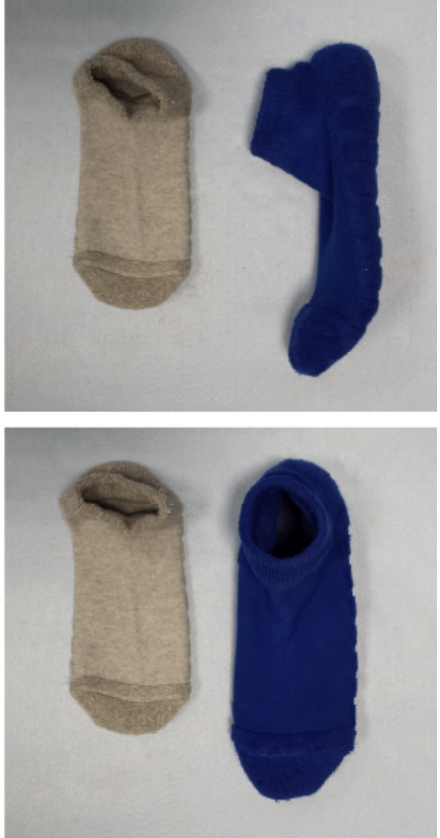

(c) After fitting

Fig. 1 Comfort-fitting shape memory sock-shoes.

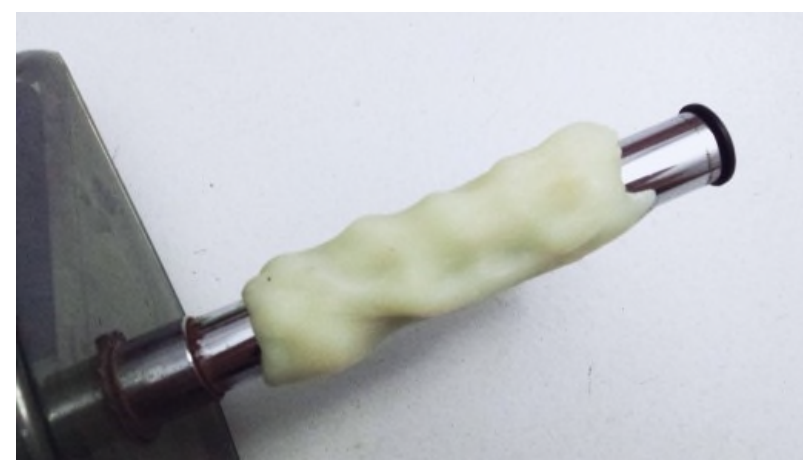

(a)

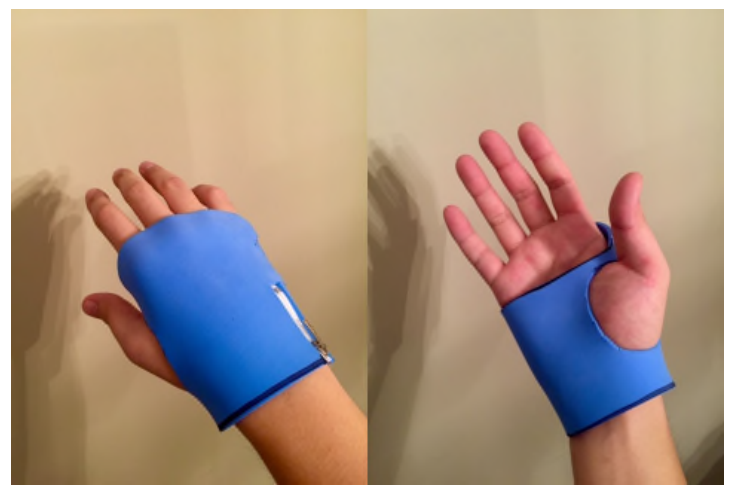

(c)

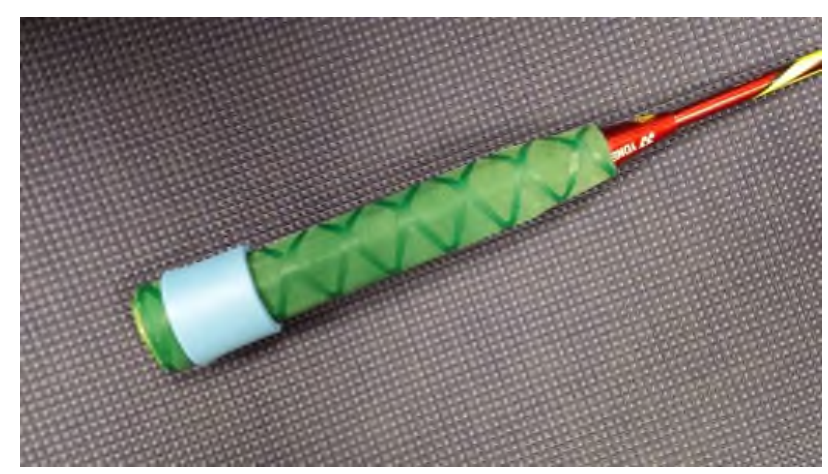

(b)

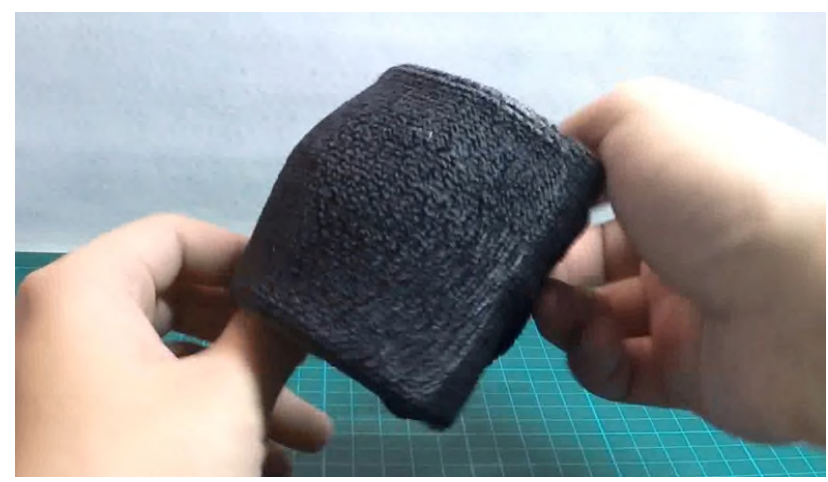

(d)

Fig. 2 Typical applications for customized fitting. (a) Handle matching individual's hand; (b) handle instantly wrapped by heat shrink tube to provide better feeling; (c) fingerless glove and (d) elbow band with instant fitting function.

\section{Acknowledgement}

The work reported here is partially supported by AcRF Tier 1 (RG172/15), Singapore and Institute 
of Sports Engineering at Nanyang Technological University, Singapore.

\section{References}

[1] S. Xiong, R. S. Goonetilleke, J. Zhao, W. Li, C. P. Witana, Foot deformations under different load-bearing conditions and their relationships to stature and body weight, Anthropol. Sci. 117(2) (2009) 77-88.

[2] M. J. Rupérez, C. Monserrat, S. Alemany, M. C. Juan, M. Alcañíz, Contact model, fit process and, foot animation for the virtual simulator of the footwear comfort, Computer-Aided Des.. 42(5) (2010) 425-431.

[3] Y. Zhou, W. M. Huang, S. F. Kang, X. L. Wu, H. B. Lu, J. Fu, et al. From 3D to 4D printing: approaches and typical applications, J. Mech. Sci. Technol. 29(10) (2015) 4281-4288.

[4] Q. Ge, C. K. Dunn, H. J. Qi, M. L. Dunn, Active origami by 4D printing, Smart Mater. Struct. 23(9) (2014) 094007.

[5] T. X. Wang, W. M. Huang, J. E. AW, L. W. He, M. WVettorello, Comfort fitting using shape memory polymeric foam, J. Polym. Eng. 45(4) (2016) Accepted.

[6] K. Otsuka, C. M. Wayman, Shape memory materials. Cambridge: Cambridge University Press; 1998.

[7] W. M. Huang, Z. Ding, C. C. Wang, J. Wei, Y. Zhao, H. Purnawali, Shape memory materials, Mater. Today, 13(7-8) (2010) 54-61.

[8] Z. G. Wei, R. Sandström, S. Miyazaki, Shape memory materials and hybrid composites for smart systems - Part II Shape-memory hybrid composites, J. Mater. Sci. 33(15) (1998) 3763-3783.

[9] Z. G. Wei, R. Sandstrom, S. Miyazaki, Shape-memory materials and hybrid composites for smart systems - Part I Shape-memory materials, J. Mater. Sci. 33(15) (1998) 3743-3762.

[10]A. Lendlein, Shape-memory Polymers: Springer-Verlag Berlin Heidelberg; 2010.

[11]L. Sun, W. M. Huang, Z. Ding, Y. Zhao, C. C. Wang, H. Purnawali, et al. Stimulus-responsive shape memory materials: a review, Mater. Des. 33 (2012) 577-640.

[12]W. M. Huang, Y. Zhao, C. C. Wang, Z. Ding, H. Purnawali, C. Tang, et al. Thermo/chemo-responsive shape memory effect in polymers: a sketch of working mechanisms, fundamentals and optimization, J. Polym. Res. 19 (2012) 9952.

[13]X. L. Wu, W. M. Huang, H. X. Tan, Characterization of shape recovery via creeping and shape memory effect in ether-vinyl acetate copolymer (EVA), J. Polym. Res. 20 (2013) 150.

[14]W. G. Yang, H. B. Lu, W. M. Huang, H. J. Qi, X. L. Wu, K. Y. Sun, Advanced shape memory technology to reshape product design, manufacturing and recycling, Polym. 6(8) (2014) 2287-2308.

[15]X. L. Wu, W. M. Huang, Z. G. Seow, W. S. Chin, W. G. Yang, K. Y. Sun, Two-step shape recovery in heating-responsive shape memory polytetrafluoroethylene and its thermally assisted self-healing, Smart Mater. Struct. 22(12) (2013) 125023.

[16]X. L. Wu, W. M. Huang, Z. Ding, H. X. Tan, W. G. Yang, K. Y. Sun, Characterization of the thermoresponsive shape-memory effect in poly(ether ether ketone) (PEEK), J. Appl. Polym. Sci. 131(3) (2014) 39844.

[17]L. Sun, W. M. Huang, C. C. Wang, Y. Zhao, Z. Ding, H. Purnawali, Optimization of the shape memory effect in shape memory polymers, J. Polym. Sci. A Polym. Chem. 49(16) (2011) 3574-3581. 
[18]W. Huang, T. Wang, Personalised footwear and the manfacture thereof. In: PCT/SG2016/050125, editor.2016. 